\title{
Existence-Uniqueness and Wright Stability Results of the Riemann-Liouville Fractional Equations by Random Controllers in MB-Spaces
}

\author{
Radko Mesiar ${ }^{1,2, *,+}$ (i) and Reza Saadati ${ }^{3, *,+(\mathbb{D})}$ \\ 1 Faculty of Civil Engineering, Slovak University of Technology, Radlinského 11, 81005 Bratislava, Slovakia \\ 2 Institute for Research and Applications of Fuzzy Modeling (IRAFM), University of Ostrava, 30. Dubna 22, \\ 70103 Ostrava 1, Czech Republic \\ 3 School of Mathematics, Iran University of Science and Technology, Narmak, Tehran 16844, Iran \\ * Correspondence: mesiar@math.sk (R.M.); rsaadati@eml.cc or rsaadati@iust.ac.ir (R.S.) \\ + These authors contributed equally to this work.
}

check for updates

Citation: Mesiar, R.; Saadati, R. Existence-Uniqueness and Wright Stability Results of the Riemann-Liouville Fractional Equations by Random Controllers in MB-Spaces. Mathematics 2021, 9, 1602. https://doi.org/10.3390/math9141602

Academic Editor: Clemente Cesarano

Received: 10 June 2021

Accepted: 29 June 2021

Published: 7 July 2021

Publisher's Note: MDPI stays neutral with regard to jurisdictional claims in published maps and institutional affiliations.

\begin{abstract}
We apply the random controllers to stabilize pseudo Riemann-Liouville fractional equations in MB-spaces and investigate existence and uniqueness of their solutions. Next, we compute the optimum error of the estimate. The mentioned process i.e., stabilization by a control function and finding an approximation for a pseudo functional equation is called random HUR stability. We use a fixed point technique derived from the alternative fixed point theorem (FPT) to investigate random HUR stability of the Riemann-Liouville fractional equations in MB-spaces. As an application, we introduce a class of random Wright control function and investigate existence-uniqueness and Wright stability of these equations in MB-spaces.
\end{abstract}

Keywords: Riemann-Liouville fractional equation; integro-differential equation; MB-spaces; wright stability; fixed point method

MSC: 46L05; 47B47; 47H10; 46L57; 39B62

\section{Introduction and Preliminaries}

We introduce the random control functions that help us to investigate existence, uniqueness, and random Wright stability of integro-differential equations in MB-spaces. Some good references for the theory and application of fractional analysis are [1-3].

We set $\mathbb{I}=[0,1], \mathbb{I}^{\circ}=(0,1), \mathbb{R}^{\bullet}=[-\infty,+\infty], \mathbb{J}^{\bullet}=[0,+\infty]$ and $\mathbb{J}^{\circ}=(0,+\infty)$. We denote the set of distribution distance mappings (DDM) by $\Sigma^{+}$. Now, $\sigma \in \Sigma^{+}$means that $\sigma$ is a mapping from $\mathbb{R}^{\bullet}$ to $\mathbb{I}$, written by $\sigma_{\tau}$ for $\sigma(\tau)$, and is left continuous and nondecreasing on $\mathbb{R}$ and also $\sigma_{0}$ is zero and $\sigma_{+\infty}=1$. Now, $S^{+} \subset \Sigma^{+}$consists of all (proper) mappings $\sigma \in \Sigma^{+}$for which $\ell^{-} \sigma_{+\infty}=1$, and $\ell^{-} \sigma_{\tau}$ means the left limit at the point $\tau$; for some more details, see [4-6]. Note that proper DDM's are the DDM's of non-negative random variables (i.e., of those random variables $g$ that a.s. take non-negative real values, $(P(\{g<0\} \cup\{g=\infty\})=0))$.

The maximal element in $\left(\Sigma^{+}, \leq\right)$is $\nabla_{\tau}^{0}$, which is defined by

$$
\nabla_{\tau}^{0}=\left\{\begin{array}{cc}
0, & \text { if } \tau \in \mathbb{R}-\mathbb{J}^{\circ}, \\
1, & \text { if } \tau \in \mathbb{J}^{\circ} .
\end{array}\right.
$$

Definition 1 ([5,7,8]). A continuous binary operation $*: \mathbb{I} \times \mathbb{I} \rightarrow \mathbb{I}$ is a CTN (continuous t-norm) whenever

(a) $\zeta * \xi=\xi * \zeta$ and $\zeta *(\kappa * \xi)=(\zeta * \kappa) * \xi$ for all $\zeta, \xi, \kappa \in \mathbb{I}$; 
(b) $\zeta * 1=\zeta$ for all $\zeta \in \mathbb{I}$;

(c) $\zeta * \kappa \leq \zeta^{\prime} * \kappa^{\prime}$ when $\zeta \leq \zeta^{\prime}$ and $\kappa \leq \kappa^{\prime}$ for every $\zeta, \zeta^{\prime}, \kappa, \kappa^{\prime} \in \mathbb{I}$.

For instance,

(1) $\quad *_{P} \kappa=\vartheta \kappa(:$ the product $\mathrm{CTN})$;

(2) $\vartheta *_{M} \kappa=\wedge\{\vartheta, \kappa\}$ (: the minimum CTN);

(3) $\vartheta *_{L} \kappa=\vee\{\vartheta+\kappa-1,0\}$ (: the Lukasiewicz CTN).

Definition 2 ([6]). Let $*$ be a CTN, $W$ be a linear space and $\zeta: W \rightarrow S^{+}$be a DDM-valued mapping. Now, $(W, \zeta, *)$ is called an $M N$-space if:

$(M N 1) \zeta_{\tau}^{w}=\nabla_{\tau}^{0}$ for every $\tau \in \mathbb{J}^{\circ}$ if and only if $w=0$;

$(M N 2) \zeta_{\tau}^{\alpha w}=\zeta_{\frac{\tau}{|\alpha|}}^{w}$ for each $w \in W$ and $\alpha \neq 0$ in $\mathbb{C}$;

$(\mathrm{MN} 3) \zeta_{\tau+\varsigma}^{w+w^{\prime}} \geq \zeta_{\tau}^{w} * \zeta_{\zeta}^{w^{\prime}}$ for every $w$ and $w^{\prime}$ in $W$ and $\tau, \varsigma \in \mathbb{J}^{\circ}$.

Here, MB-space represents a complete MN-space $[9,10]$. In the following, we suppose that $*=*_{M}$.

Theorem 1 ([11,12]). Consider the complete $\mathbb{J}^{\bullet}$-valued metric space $(S, \delta)$ and also consider the self-map $\Lambda$ on $S$ such that

$$
\delta(\Lambda s, \Lambda t) \leq \kappa \delta(t, s), \kappa<1 \text {, where } \kappa \text { is a Lipschitz constant }
$$

Assume that $s \in S$, so there are two options:

$$
\delta\left(\Lambda^{m} s, \Lambda^{m+1} s\right)=\infty, \forall m \in \mathbb{N},
$$

or

(II) there is $m_{0} \in \mathbb{N}$ such that

(1) $\delta\left(\Lambda^{m} s, \Lambda^{m+1} s\right)<\infty, \quad \forall m \geq m_{0}$;

(2) the sequence $\Lambda^{m}$ s converges to a fixed point $t^{*}$ of $\Lambda$;

(3) $t^{*} \in V=\left\{t \in S \mid \delta\left(\Lambda^{m_{0}} s, t\right)<\infty\right\}$ is the unique fixed point of $\Lambda$ in $V$;

(4) $(1-\kappa) \delta\left(t, t^{*}\right) \leq \delta(t, \Lambda t)$ for every $t \in V$.

\section{Riemann-Liouville Fractional Equations}

Let $u:[p, q] \rightarrow \mathbb{R}(0<p<q<\infty)$ be a continuous function and $\varrho>0$ a real number. We define the Riemann-Liouville fractional integrals of order $\varrho$, by

$$
{ }_{p} \mathcal{I}_{s}^{\varrho} u(s)=\frac{1}{\Gamma(\varrho)} \int_{p}^{s}(s-\sigma)^{\varrho-1} u(\sigma) d \sigma, \quad p<s .
$$

Using the definition of Riemann-Liouville fractional integrals, we define the RiemannLiouville derivatives as follows:

$$
\begin{aligned}
{ }_{p} \mathcal{D}_{s}^{\varrho} u(s) & =\left(\frac{d}{d s}\right)^{k}{ }_{p} \mathcal{I}_{s}^{k-\varrho} u(s) \\
& =\frac{1}{\Gamma(k-\varrho)}\left(\frac{d}{d s}\right)^{k} \int_{p}^{s}(s-\sigma)^{k-\varrho-1} u(\sigma) d \sigma, \quad p<s, \quad k-1<\varrho<k .
\end{aligned}
$$

Let $T$ be a real positive number. Consider the Riemann-Liouville fractional Volterra integro-differential equation, defined by

$$
{ }_{0} \mathcal{D}_{s}^{\varrho} u(s)=\alpha(s, u(s))+\int_{0}^{s} \mathcal{K}(s, \sigma, u(\sigma)) d \sigma
$$

where $\varrho \in \mathbb{I}^{\circ}, \alpha:[0, T] \times W \rightarrow W, \mathcal{K}:[0, T] \times[0, T] \times W \rightarrow W$. 
In [13], Goleț, defined the concept of differentiable functions in an MB-space $(W, \zeta, *)$ and proved that, if the function $f: U \rightarrow(W, \zeta, *)$ is differentiable in $u_{0} \in U$, it is therefore continuous in the point $u_{0}$.

The Wright function $[14,15]$ is one of the special functions defined by the series representation, valid in the whole complex plane

$$
W_{\alpha, \beta}(z)=\sum_{n=0}^{\infty} \frac{z^{n}}{n ! \Gamma(n \alpha+\beta)}
$$

where $\alpha>-1$ and $\beta \in \mathbb{C}$. In this paper, we define a distribution distance mapping (DDM) based on Wright functions.

Consider the DDM-valued $\mathbf{W}_{\alpha, \beta}:[0, T] \rightarrow S^{+}\left(\alpha, \beta \in \mathbb{J}^{\circ}\right)$, a random control mapping, which is defined as follows:

$$
\left(\mathbf{W}_{\alpha, \beta}\right)_{\tau}^{S}=\left\{\begin{array}{cc}
0, & \text { if } \tau \in \mathbb{R}-\mathbb{J}^{\circ}, \\
\sum_{n=0}^{\infty} \frac{\left(\frac{-\|s\|}{\tau}\right)^{n}}{n ! \Gamma(n \alpha+\beta)}, & \text { if } \tau \in \mathbb{J}^{\circ}
\end{array}\right.
$$

Then, we have

- It is left continuous and always increasing for positive values, it means that, for $\tau>0,\left(\mathbf{W}_{\alpha, \beta}\right)_{\tau}^{s}>0$

- $\lim _{\tau \longrightarrow \infty}\left(\mathbf{W}_{\alpha, \beta}\right)_{\tau}^{S}=1$

- $\quad$ For $\tau \leq 0$, we have $\left(\mathbf{W}_{\alpha, \beta}\right)_{\tau}^{s}=0$,

And the following conditions also apply to the DDM-valued Wright function

(MN1) We show that $\left(\mathbf{W}_{\alpha, \beta}\right)_{\tau}^{s}=1$ iff $s=0$.

$$
\begin{aligned}
& \left(\mathbf{W}_{\alpha, \beta}\right)_{\tau}^{s}=\sum_{n=0}^{\infty} \frac{\left(\frac{-\|s\|}{\tau}\right)^{n}}{n ! \Gamma(n \alpha+\beta)}=1+\sum_{n=1}^{\infty} \frac{\left(\frac{-\|s\|}{\tau}\right)^{n}}{n ! \Gamma(n \alpha+\beta)}=1 \\
& \Longrightarrow \sum_{n=1}^{\infty} \frac{\left(\frac{-\|s\|}{\tau}\right)^{n}}{n ! \Gamma(n \alpha+\beta)}=0 \Longrightarrow\|s\|=0 \Longrightarrow s=0
\end{aligned}
$$

and vice versa with $s=0$.

(MN2)

$$
\left(\mathbf{W}_{\alpha, \beta}\right)_{\tau}^{\alpha s}=\sum_{n=0}^{\infty} \frac{\left(\frac{-\|\alpha s\|}{\tau}\right)^{n}}{n ! \Gamma(n \alpha+\beta)}=\sum_{n=0}^{\infty} \frac{\left(\frac{-|\alpha|\|s\|}{\tau}\right)^{n}}{n ! \Gamma(n \alpha+\beta)}=\sum_{n=0}^{\infty} \frac{\left(\frac{-\|s\|}{\frac{\tau}{|\alpha|}}\right)^{n}}{n ! \Gamma(n \alpha+\beta)}=\left(\mathbf{W}_{\alpha, \beta}\right)_{\frac{\tau}{|\alpha|}}^{s}
$$

(MN3) We assume that

$$
\sum_{n=0}^{\infty} \frac{\left(\frac{-\|s\|}{\tau}\right)^{n}}{n ! \Gamma(n \alpha+\beta)} \leq \sum_{n=0}^{\infty} \frac{\left(\frac{-\left\|s^{\prime}\right\|}{\varsigma}\right)^{n}}{n ! \Gamma(n \alpha+\beta)}
$$

Thus,

$$
\begin{aligned}
& \frac{-\|s\|}{\tau} \leq \frac{-\left\|s^{\prime}\right\|}{\varsigma} \Longrightarrow \frac{\|s\|}{\tau} \geq \frac{\left\|s^{\prime}\right\|}{\varsigma} \Longrightarrow \frac{\varsigma\|s\|}{\tau} \geq\left\|s^{\prime}\right\| \Longrightarrow \frac{s\|s\|}{\tau}+\|s\| \\
& \geq\left\|s^{\prime}\right\|+\|s\| \geq\left\|s+s^{\prime}\right\| \Longrightarrow\|s\|\left(\frac{s}{\tau}\right) \geq\left\|s+s^{\prime}\right\| \Longrightarrow \frac{s+\tau}{\tau}\|s\| \geq\left\|s+s^{\prime}\right\| \Longrightarrow \frac{\|s\|}{\tau} \\
& \geq \frac{\left\|s+s^{\prime}\right\|}{\tau+\varsigma} \Longrightarrow \frac{-\|s\|}{\tau} \leq \frac{-\left\|s+s^{\prime}\right\|}{\tau+s} \Longrightarrow \sum_{n=0}^{\infty} \frac{\left(\frac{-\left\|s+s^{\prime}\right\|}{\tau+\varsigma}\right)^{n}}{n ! \Gamma(n \alpha+\beta)} \geq \sum_{n=0}^{\infty} \frac{\left(\frac{-\|s\|}{\tau}\right)^{n}}{n ! \Gamma(n \alpha+\beta)} .
\end{aligned}
$$

Now, we conclude that

$$
\left(\mathbf{W}_{\alpha, \beta}\right)_{\tau+\varsigma}^{s+s^{\prime}} \geq\left(\mathbf{W}_{\alpha, \beta}\right)_{\tau}^{S} *_{M}\left(\mathbf{W}_{\alpha, \beta}\right)_{\zeta}^{s^{\prime}}
$$


Let DDM-valued $\psi:[0, T] \rightarrow S^{+}$be a random control mapping. We say that (3) is random HUR stable, when, for a differentiable mapping $u(s)$, satisfying

$$
\zeta_{\tau}^{0} \mathcal{D}_{s}^{\varrho} u(s)-\alpha(s, u(s))-\int_{0}^{s} \mathcal{K}(s, \sigma, u(\sigma)) d \sigma \geq \psi_{\tau}^{s},
$$

$s \in[0, T]$, there is a solution $v(s)$ of Equation (3) such that, for some $r>0$,

$$
\zeta_{\tau}^{u(s)-v(s)} \geq \psi_{\frac{\tau}{r}}^{s} .
$$

If we replace the control function $\psi$ with the DDM-valued Wright function $\left(\mathbf{W}_{\alpha, \beta}\right)$, we say that (3) is random Wright stable.

\section{Riemann-Liouville Fractional Volterra Integro-Differential Equation}

In 2008, Mihet and Radu [16,17] introduced a new method to investigate random stability in MB-spaces and then some authors used this method to get stability results for new equations [18-30]. Here, we use the Mihet and Radu method and Theorem 1 to investigate random Wright stability of (3) and improve recent results [31]; we can suggest [32-34] for more details. We set

$$
B:=\{u:[0, T] \rightarrow W, u \text { is differentiable }\}
$$

and consider $\delta$ from $B \times B$ to $\mathbb{J}^{\bullet}$ by

$\delta(u, v)=\inf \left\{\mu \in \mathbb{J}^{\circ}: \zeta_{\tau}^{0} \mathcal{D}_{s}^{o} u(s){ }_{0} \mathcal{D}_{s}^{o} v(s) * \zeta_{\tau}^{u(s)-v(s)} \geq \psi_{\frac{\tau}{\mu}}^{s}, \forall u, v \in B, s \in[0, T], \tau \in \mathbb{J}^{\circ}\right\}$.

Theorem 2. $\mathbb{J}^{\bullet}$-valued metric space $(B, \delta)$ is complete.

Proof. Letting $\delta(u, v)=0$, we have

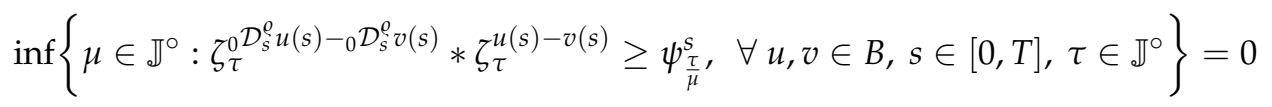

and so

$$
\zeta_{\tau}^{0} \mathcal{D}_{s}^{\rho} u(s)-{ }_{0} \mathcal{D}_{s}^{\rho} v(s) * \zeta_{\tau}^{u(s)-v(s)} \geq \psi_{\frac{\tau}{\mu}}^{s},
$$

for all $\mu \in \mathbb{J}^{\circ}$. Tend $\mu$ to zero in the above inequality, we get

$$
\zeta_{\tau}^{0} \mathcal{D}_{s}^{\rho} u(s)-{ }_{0} \mathcal{D}_{s}^{\rho} v(s) * \zeta_{\tau}^{u(s)-v(s)}=\nabla_{\tau}^{0}
$$

and so

$$
\zeta_{\tau}^{u(s)-v(s)}=\nabla_{\tau}^{0}
$$

thus, $u(s)=v(s)$ for every $s \in[0, T]$, and vice versa. In addition, we have $\delta(u, v)=\delta(v, u)$ for every $u, v \in B$. Now, let $\delta(u, v)=e_{1} \in \mathbb{J}^{\circ}$ and $\delta(v, w)=e_{2} \in \mathbb{J}^{\circ}$. Thus, we have

$$
\zeta_{\tau}^{0} \mathcal{D}_{s}^{\rho} u(s)-{ }_{0} \mathcal{D}_{s}^{\varrho} v(s) * \zeta_{\tau}^{u(s)-v(s)} \geq \psi_{\frac{\tau}{e_{1}}}^{s}
$$

and

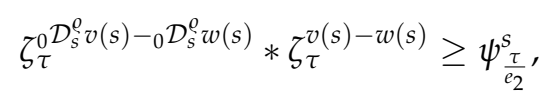


for every $\tau \in \mathbb{J}^{\circ}$. Thus, we have

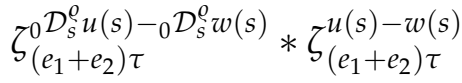

$$
\begin{aligned}
& \geq\left[\zeta_{e_{1} \tau}^{0} \mathcal{D}_{s}^{\rho} u(s)-{ }_{0} \mathcal{D}_{s}^{\rho} v(s) * \zeta_{e_{2} \tau}^{0} \mathcal{D}_{s}^{\rho} v(s)-{ }_{0} \mathcal{D}_{s}^{\rho} w(s)\right] *\left[\zeta_{e_{1} \tau}^{u(s)-v(s)} * \zeta_{e_{2} \tau}^{v(s)-w(s)}\right] \\
& \geq \psi_{\tau}^{s} * \psi_{\tau}^{s} \\
& \geq \psi_{\tau}^{S}
\end{aligned}
$$

and so $\delta(u, w) \leq e_{1}+e_{2}$. Thus, $\delta(u, w) \leq \delta(u, v)+\delta(v, w)$. To show completeness of $(B, \delta)$, we consider the Cauchy sequence $\left\{u_{k}\right\}_{k}$ in $(B, \delta)$. Suppose that $s \in[0, T]$ is fixed. Assume that $\omega \in \mathbb{J}^{\circ}$ and $\lambda \in \mathbb{I}^{\circ}$ are arbitrary and consider $\tau \in \mathbb{J}^{\circ}$ such that $\theta_{\tau}^{s}>1-\lambda$. For $e \tau<\omega$, choose $k_{0} \in \mathbb{N}$ such that

$$
\delta\left(u_{k}-u_{\ell}\right)<e \forall k, \ell \geq k_{0} .
$$

Consequently,

$$
\begin{aligned}
& \zeta_{\omega}^{0} \mathcal{D}_{s}^{\rho} u_{k}(s)-{ }_{0} \mathcal{D}_{s}^{\rho} u_{\ell}(s) * \zeta_{\omega}^{u_{k}(s)-u_{\ell}(s)} \\
& \geq \zeta_{e \tau}^{0} \mathcal{D}_{s}^{\rho} u_{k}(s)-{ }_{0} \mathcal{D}_{s}^{\rho} u_{\ell}(s) * \zeta_{e \tau}^{u_{k}(s)-u_{\ell}(s)} \\
& \geq \psi_{\tau}^{s} \\
& >1-\lambda \text {. }
\end{aligned}
$$

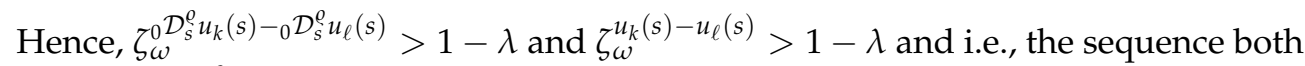
$\left\{u_{k}(s)\right\}_{k}$ and $\left\{{ }_{0} \mathcal{D}_{s}^{\varrho} u_{k}(s)\right\}_{k}$ are Cauchy in complete space $(W, \zeta, *)$ on compact set $[0, T]$, so they are uniformly convergent to the mapping $u:[0, T] \rightarrow W$ and ${ }_{0} \mathcal{D}_{s}^{\varrho} u$, respectively. Now, if we apply the uniform convergence, we conclude that $u \in B$ and is differentiable; thus, $(B, \delta)$ is complete.

Theorem 3. Let $(W, \zeta, *)$ be an $M B$-space and $\ell_{1}, \ell_{2}, \ell_{3}, \ell_{4}$ and $T$ be a positive constant such that $\bigvee\left\{\ell_{1}, \ell_{2} \ell_{3}, \ell_{1} \ell_{4}, \ell_{2} \ell_{3} \ell_{4}\right\}<0.5$. Assume that the continuous mappings $\alpha:[0, T] \times W \rightarrow W$, $\mathcal{K}:[0, T] \times[0, T] \times W \rightarrow W$ with DDM-valued $\psi:[0, T] \rightarrow S^{+}$satisfying

$$
\begin{aligned}
\zeta_{\tau}^{\alpha(s, u(s))-\alpha(s, v(s))} \geq \zeta_{\frac{\tau}{\ell_{1}}}^{u(s)-v(s)}, \\
\zeta_{\tau}^{\mathcal{K}(s, \sigma, u(\sigma))-\mathcal{K}(s, \sigma, v(\sigma))} \geq \zeta_{\frac{\tau}{\ell_{2}}}^{u(\sigma)-v(\sigma)}, \sigma \leq s, \\
\inf _{\rho \in[0, T]} \psi_{\tau}^{\rho} \geq \psi_{\frac{T \tau}{\ell_{3}}}^{s},
\end{aligned}
$$

and

$$
\zeta_{\tau}^{u(s)} \geq \psi_{\tau}^{s}, \text { implies that } \zeta_{\tau}^{0 \mathcal{I}_{s}^{\rho} u(\sigma) d \sigma} \geq \psi_{\frac{\tau}{\ell_{4}}}^{s},
$$

for every $s \in[0, T], u, v:[0, T] \rightarrow W$ and $\tau \in \mathbb{J}^{\circ}$. Let $w:[0, T] \rightarrow W$ be a differentiable function satisfying

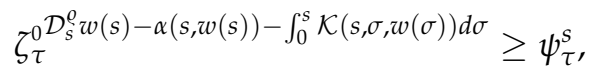

for every $s \in[0, T]$ and $\tau \in \mathbb{J}^{\circ}$. Thus, there is a unique differentiable function $w_{0}:[0, T] \rightarrow W$ such that

$$
{ }_{0} \mathcal{D}_{s}^{\varrho} w_{0}(s)=\alpha\left(s, w_{0}(s)\right)+\int_{0}^{s} \mathcal{K}\left(s, \sigma, w_{0}(\sigma)\right) d \sigma,
$$


and

$$
\zeta_{\tau}^{0} \mathcal{D}_{s}^{\rho} w(s)-{ }_{0} \mathcal{D}_{s}^{\rho} w_{0}(s) * \zeta_{\tau}^{w(s)-w_{0}(s)} \geq \frac{\psi_{\left(1-2 \bigvee\left\{\ell_{1}, \ell_{2} \ell_{3}, \ell_{1} \ell_{4}, \ell_{2} \ell_{3} \ell_{4}\right\}\right) \tau}^{S}}{\bigvee\left\{1, \ell_{4}\right\}}
$$

for every $s \in[0, T]$ and $\tau \in \mathbb{J}^{\circ}$.

Proof. We set

$$
B:=\{u:[0, T] \rightarrow W, u \text { is differentiable }\}
$$

and define

$$
\delta(u, v)=\inf \left\{\mu \in \mathbb{J}^{\circ}: \zeta_{\tau}^{0} \mathcal{D}_{s}^{\varrho} u(s){ }_{0} \mathcal{D}_{s}^{\varrho} v(s) * \zeta_{\tau}^{u(s)-v(s)} \geq \psi_{\frac{\tau}{\mu}}^{s}, \forall u, v \in B, s \in[0, T], \tau \in \mathbb{J}^{\circ}\right\} .
$$

Theorem 2 guarantees that $(B, \delta)$ is a complete $\mathbb{J}^{\bullet}$-valued metric space.

Consider the self-map $Y$ on $B$ by

$$
\mathrm{Y}(u(s))={ }_{0} \mathcal{I}_{s}^{\varrho}(\alpha(\sigma, u(\sigma)))+{ }_{0} \mathcal{I}_{s}^{\varrho}\left(\int_{0}^{\sigma} \mathcal{K}(\sigma, \varsigma, u(\varsigma)) d \varsigma\right),
$$

where $\varrho \in \mathbb{I}^{\circ}, \alpha:[0, T] \times W \rightarrow W, \mathcal{K}:[0, T] \times[0, T] \times W \rightarrow W$. We prove that $Y$ is a strictly contractive mapping. Let $u, v \in B, \mu \in \mathbb{J}^{\circ}$ and $\delta(u, v)<v$; thus, we have

$$
\zeta_{v \tau}^{0} \mathcal{D}_{\xi}^{\rho} u(s)-{ }_{0} \mathcal{D}_{s}^{\rho} v(s) * \zeta_{v \tau}^{u(s)-v(s)} \geq \psi_{\tau}^{s}, \quad \forall u, v \in B, s \in[0, T], \tau \in \mathbb{J}^{\circ} .
$$

Using properties (MN2) and (MN3) of Definition 2 and (11), imply that

$$
\begin{aligned}
& \zeta_{2 v \tau}^{0} \mathcal{D}_{s}^{\rho} \mathrm{Y}(u(s)){ }_{0} \mathcal{D}_{s}^{\rho} \mathrm{Y}(v(s)) * \zeta_{2 v \tau}^{\mathrm{Y}(u(s))-\mathrm{Y}(v(s))} \\
& =\zeta_{2 v \tau}^{[\alpha(s, u(s))-\alpha(s, v(s))]+\int_{0}^{s}[\mathcal{K}(s, \sigma, u(\sigma))-\mathcal{K}(s, \sigma, v(\sigma))] d \sigma} \\
& \text { * } \quad \zeta_{2 v \tau}^{0 \mathcal{I}_{s}^{Q}(\alpha(s, u(s))-\alpha(s, v(s)))+{ }_{0} \mathcal{I}_{s}^{Q}\left(\int_{0}^{s}[\mathcal{K}(s, \sigma, u(\sigma))-\mathcal{K}(s, \sigma, v(\sigma))] d \sigma\right)} \\
& \geq \zeta_{v \tau}^{\alpha(s, u(s))-\alpha(s, v(s))} * \zeta_{v \tau}^{\int_{0}^{s}[\mathcal{K}(s, \sigma, u(\sigma))-\mathcal{K}(s, \sigma, v(\sigma))] d \sigma} \\
& * \quad \zeta_{v \tau}^{0} \mathcal{I}_{s}^{\varrho}(\alpha(\sigma, u(\sigma))-\alpha(\sigma, v(\sigma))) * \zeta_{v \tau}^{0} \mathcal{I}_{s}^{Q}\left(\int_{0}^{\sigma}[\mathcal{K}(\sigma, \zeta, u(\varsigma))-\mathcal{K}(\sigma, \zeta, v(\varsigma))] d \zeta\right) .
\end{aligned}
$$

Now, we connect (12) and control function $\psi$. Assume that $0=\omega_{1}<\omega_{2}<\cdots<$ $\omega_{k}=s, \Delta \omega_{i}=\omega_{i}-\omega_{i-1}=\frac{s}{k}, i=1,2, \cdots, k$ and $\|\Delta \omega\|=\bigvee_{1 \leq i \leq k}\left(\Delta \omega_{i}\right)$.

Step 1. From (35), we have

$$
\begin{aligned}
\zeta_{v \tau}^{\alpha(s, u(s))-\alpha(s, v(s))} & \geq \zeta_{\frac{v \tau}{\ell_{1}}}^{u(s)-v(s)} \\
& \geq \psi_{\frac{\tau}{\ell_{1}}}^{s}
\end{aligned}
$$


Step 2. Using (MN2) and (MN3) of Definition 2, continuity property of DDM-valued $\zeta,(36)$, and (37), we get

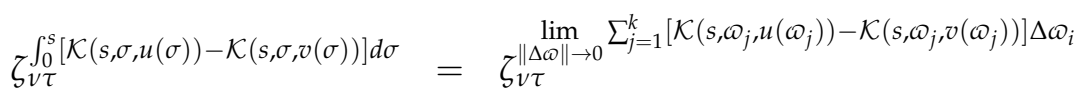

$$
\begin{aligned}
& =\lim _{\|\Delta \omega\| \rightarrow 0} \zeta_{v \tau}^{\sum_{j=1}^{k}\left[\mathcal{K}\left(s, \omega_{j}, u\left(\omega_{j}\right)\right)-\mathcal{K}\left(s, \omega_{j}, v\left(\omega_{j}\right)\right)\right] \Delta \omega_{i}} \\
& \geq \lim _{\|\Delta \omega\| \rightarrow 0} \bigwedge_{j=1}^{k} \zeta_{\frac{v \tau}{k}}^{\left[\mathcal{K}\left(s, \omega_{j}, u\left(\omega_{j}\right)\right)-\mathcal{K}\left(s, \omega_{j}, v\left(\omega_{j}\right)\right)\right] \Delta \omega_{i}} \\
& \geq \inf _{\rho \in[0, T]} \zeta_{\frac{k v \tau}{k T}}^{\mathcal{K}(s, \rho, u(\rho))-\mathcal{K}(s, \rho, v(\rho))} \\
& \geq \inf _{\rho \in[0, T]} \zeta_{\frac{k v \tau}{k T \ell_{2}}}^{u(\rho)-v(\rho)} \\
& \geq \inf _{\rho \in[0, T]} \psi_{\frac{\tau}{\ell_{2} T}}^{\rho} \\
& \geq \psi_{\frac{\tau}{\ell_{2} \ell_{3}}}^{S} \text {. }
\end{aligned}
$$

Step 3. Using (38) and (13), we get

$$
\zeta_{v \tau}^{0 \mathcal{I}_{s}^{\varrho}(\alpha(\sigma, u(\sigma))-\alpha(\sigma, v(\sigma)))} \geq \psi_{\frac{\tau}{\ell_{1} \ell_{4}}}^{S} .
$$

Step 4. Using (38) and (14), we get

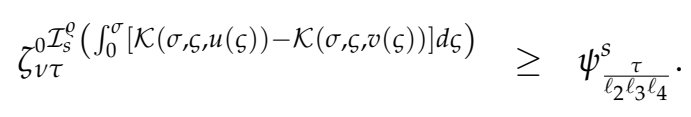

Form (12)-(16), we have

$$
\begin{aligned}
& \zeta_{2 v \tau}^{0} \mathcal{D}_{S}^{\varrho} \mathrm{Y}(u(s))-{ }_{0} \mathcal{D}_{s}^{\varrho} \mathrm{Y}(v(s)) * \zeta_{2 v \tau}^{\mathrm{Y}(u(s))-\mathrm{Y}(v(s))} \\
& \geq \psi_{\frac{\tau}{\ell_{1}}}^{S} * \psi_{\frac{\tau}{\ell_{2} \ell_{3}}}^{S} * \psi_{\frac{\tau}{\ell_{1} \ell_{4}}}^{S} * \psi_{\frac{\tau}{\ell_{2} \ell_{3} \ell_{4}}}^{S} \\
& \geq \psi_{\frac{S}{\mathrm{~S}}} \frac{\tau}{\nabla\left\{\ell_{1}, \ell_{2} \ell_{3}, \ell_{1} \ell_{4}, \ell_{2} \ell_{3} \ell_{4}\right\}}
\end{aligned}
$$

and so

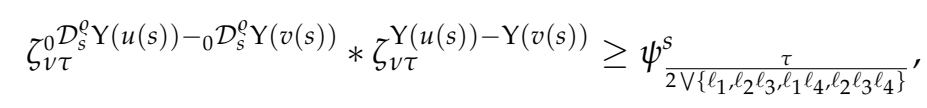

which implies that

$$
\delta(\mathrm{Y}(u), \mathrm{Y}(v)) \leq 2 \bigvee\left\{\ell_{1}, \ell_{2} \ell_{3}, \ell_{1} \ell_{4}, \ell_{2} \ell_{3} \ell_{4}\right\} v
$$

and so

$$
\delta(\mathrm{Y}(u), \mathrm{Y}(v)) \leq 2 \bigvee\left\{\ell_{1}, \ell_{2} \ell_{3}, \ell_{1} \ell_{4}, \ell_{2} \ell_{3} \ell_{4}\right\} \delta(u, v)
$$

Consequently, $\mathrm{Y}$ is a strictly contractive mapping with Lipschitz constant

$$
2 \bigvee\left\{\ell_{1}, \ell_{2} \ell_{3}, \ell_{1} \ell_{4}, \ell_{2} \ell_{3} \ell_{4}\right\}
$$

Letting $w \in B$, we show that $\delta(\mathrm{Y}(w), w)<\infty$. Using (38) and (39), we get 


$$
\begin{aligned}
& \zeta_{\tau}^{0} \mathcal{D}_{s}^{\rho}[\mathrm{Y}(w(s))-w(s)] * \zeta_{\tau}^{\mathrm{Y}(w(s))-w(s)} \\
& =\zeta_{\tau}^{\alpha(s, w(s))+\int_{0}^{s} \mathcal{K}(s, \sigma, w(\sigma)) d \sigma-{ }_{0} \mathcal{D}_{s}^{\rho} w(s)} * \zeta_{\tau}^{0} \mathcal{I}_{s}^{\rho}(\alpha(\sigma, w(\sigma)))+{ }_{0} \mathcal{I}_{s}^{\varrho}\left(\int_{0}^{\sigma} \mathcal{K}(\sigma, \zeta, w(\varsigma)) d \varsigma\right)-{ }_{0} \mathcal{I}_{s}^{\varrho} \mathcal{D}_{\sigma}^{\varrho} w(\sigma) \\
& =\zeta_{\tau}^{\alpha(s, w(s))+\int_{0}^{s} \mathcal{K}(s, \sigma, w(\sigma)) d \sigma-{ }_{0} \mathcal{D}_{s}^{\varrho} w(s)} * \zeta_{\tau}^{0} \mathcal{I}_{s}^{\varrho}\left[(\alpha(\sigma, w(\sigma)))+\left(\int_{0}^{\sigma} \mathcal{K}(\sigma, \zeta, w(\varsigma)) d \varsigma\right)-{ }_{0} \mathcal{D}_{\sigma}^{\varrho} w(\sigma)\right] \\
& \geq \psi_{\tau}^{S} * \psi_{\frac{\tau}{\ell_{4}}}^{S} \\
& \geq \psi_{\frac{\tau}{\mathrm{V}\left\{1, \ell_{4}\right\}}}{ }^{\prime}
\end{aligned}
$$

for every $\tau \in \mathbb{J}^{\circ}$. Then, we have $\delta(Y(w), w)<\bigvee\left\{1, \ell_{4}\right\}<\infty$.

By Theorem 1 , there is $w_{0}$ in $B$ such that

(1) $w_{0}$ is a fixed point of $Y$, i.e.,

$$
\begin{aligned}
w_{0}(s) & =\mathrm{Y}\left(w_{0}(s)\right) \\
& ={ }_{0} \mathcal{I}_{s}^{\varrho}\left(\alpha\left(\sigma, w_{0}(\sigma)\right)\right)+{ }_{0} \mathcal{I}_{s}^{\varrho}\left(\int_{0}^{\sigma} \mathcal{K}\left(\sigma, \varsigma, w_{0}(\varsigma)\right) d \varsigma\right),
\end{aligned}
$$

which is unique in

$$
B^{*}=\{u \in B: \delta(\mathrm{Y}(w), u)<\infty\} .
$$

Taking ${ }_{0} \mathcal{D}_{S}^{\varrho}$ from (22), we get

$$
{ }_{0} \mathcal{D}_{s}^{\varrho} w_{0}(s)=\alpha\left(s, w_{0}(s)\right)+\int_{0}^{s} \mathcal{K}\left(s, \sigma, w_{0}(\sigma)\right) d \sigma,
$$

where $\varrho \in \mathbb{I}^{\circ}, \alpha:[0, T] \times W \rightarrow W, \mathcal{K}:[0, T] \times[0, T] \times W \rightarrow W$.

(2) $\delta\left(Y^{k}(w), w_{0}\right) \rightarrow 0$ as $n \rightarrow \infty$; plies that

(3) $\delta\left(w, w_{0}\right) \leq \frac{1}{1-2 \bigvee\left\{\ell_{1}, \ell_{2} \ell_{3}, \ell_{1} \ell_{4}, \ell_{2} \ell_{3} \ell_{4}\right\}} \delta(\mathrm{Y}(w), w) \leq \frac{\bigvee\left\{1, \ell_{4}\right\}}{1-2 \bigvee\left\{\ell_{1}, \ell_{2} \ell_{3}, \ell_{1} \ell_{4}, \ell_{2} \ell_{3} \ell_{4}\right\}}$, which im-

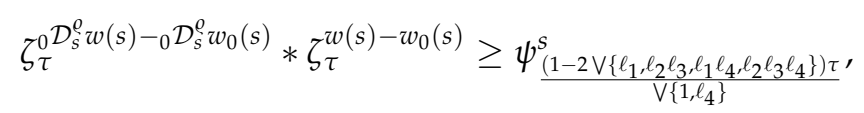

for every $s \in[0, T]$ and $\tau \in \mathbb{J}^{\circ}$.

Now, we prove that $B^{*}=B$. Considering $z_{0}$ in $B$ satisfying (40) and (41), we show that $z_{0}=w_{0}$ and $z_{0} \in B^{*}$. From (40), we get

$$
{ }_{0} \mathcal{D}_{s}^{\varrho} z_{0}(s)=\alpha\left(s, z_{0}(s)\right)+\int_{0}^{s} \mathcal{K}\left(s, \sigma, z_{0}(\sigma)\right) d \sigma,
$$

and so

$$
\begin{aligned}
z_{0}(s) & ={ }_{0} \mathcal{I}_{s}^{\varrho} \alpha\left(\sigma, z_{0}(\sigma)\right)+{ }_{0} \mathcal{I}_{s}^{\varrho} \int_{0}^{\sigma} \mathcal{K}\left(\sigma, \varsigma, z_{0}(\varsigma)\right) d \varsigma \\
& =\mathrm{Y}\left(z_{0}(s)\right),
\end{aligned}
$$

where $\varrho \in \mathbb{I}^{\circ}, \alpha:[0, T] \times W \rightarrow W, \mathcal{K}:[0, T] \times[0, T] \times W \rightarrow W$.

Now, we show that

$$
z_{0} \in\{u \in B: \delta(\mathrm{Y}(w), u)<\infty\}
$$

i.e., $\delta\left(Y(w), z_{0}\right)<\infty$. We set $]=\frac{1-2 \bigvee\left\{\ell_{1}, \ell_{2} \ell_{3}, \ell_{1} \ell_{4}, \ell_{2} \ell_{3} \ell_{4}\right\}}{\bigvee\left\{1, \ell_{4}\right\}}$, from (41), we get

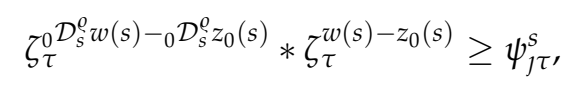

for every $s \in[0, T]$ and $\tau \in \mathbb{J}^{\circ}$. 
Form (35) and (27), we get

$$
\begin{aligned}
\zeta_{\tau}^{\alpha(s, w(s))-\alpha\left(s, z_{0}(s)\right)} & \geq \zeta_{\frac{\tau}{\ell_{1}}}^{w(s)-z_{0}(s)} \\
& \geq \psi_{j \frac{\tau}{\ell_{1}}}^{s} ;
\end{aligned}
$$

in addition, from (36) and (27), we get

$$
\begin{aligned}
\zeta_{\tau}^{\mathcal{K}(s, \sigma, w(\sigma))-\mathcal{K}\left(s, \sigma, z_{0}(\sigma)\right)} & \geq \zeta_{\frac{\tau}{\ell_{2}}}^{w(\sigma)-z_{0}(\sigma)} \\
& \geq \psi_{j \frac{\tau}{\ell_{2}}}^{s}
\end{aligned}
$$

for every $s \in[0, T], \sigma \leq s$ and $\tau \in \mathbb{J}^{\circ}$. By step 2 and (29), we get

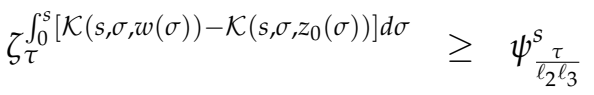

$$
\begin{aligned}
& \geq \psi_{j \frac{\tau}{\ell_{2} \ell_{3}}}^{s}
\end{aligned}
$$

Using triangular inequality (MN3), (28) and (30), we get

$$
\begin{aligned}
& \zeta_{2 \tau}^{\alpha(s, w(s))-\alpha\left(s, z_{0}(s)\right)+\int_{0}^{s}\left[\mathcal{K}(s, \sigma, w(\sigma))-\mathcal{K}\left(s, \sigma, z_{0}(\sigma)\right)\right] d \sigma} \\
& \geq \zeta_{\tau}^{\alpha(s, w(s))-\alpha\left(s, z_{0}(s)\right)} * \zeta_{\tau}^{\int_{\tau}^{s}}\left[\mathcal{K}(s, \sigma, w(\sigma))-\mathcal{K}\left(s, \sigma, z_{0}(\sigma)\right)\right] d \sigma \\
& \geq \psi_{j \frac{\tau}{\ell_{1}}}^{s} * \psi_{j \frac{\tau}{\ell_{2} \ell_{3}}}^{s} \\
& \geq \psi_{J}^{s} \frac{\tau}{V\left\{\ell_{1}, \ell_{2} \ell_{3}\right\}},
\end{aligned}
$$

and so

$$
\zeta_{\tau}^{\alpha(s, w(s))-\alpha\left(s, z_{0}(s)\right)+\int_{0}^{s}\left[\mathcal{K}(s, \sigma, w(\sigma))-\mathcal{K}\left(s, \sigma, z_{0}(\sigma)\right)\right] d \sigma} \geq \psi_{\frac{J_{j}}{2 \bigvee\left\{\ell_{1}, \ell_{2} \ell_{3}\right\}}} .
$$

We apply (38) and get

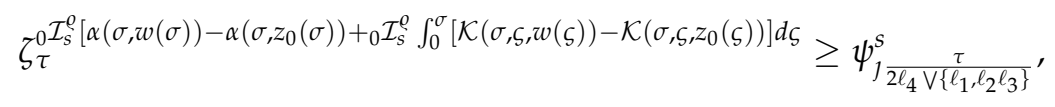

for every $s \in[0, T], \sigma \leq s$ and $\tau \in \mathbb{J}^{\circ}$.

Using (32), (32), and (33), we get

$$
\begin{aligned}
& \zeta_{\tau}^{0} \mathcal{D}_{s}^{\varrho}\left[\mathrm{Y}(w(s))-z_{0}(s)\right] * \zeta_{\tau}^{\mathrm{Y}(w(s))-z_{0}(s)}
\end{aligned}
$$

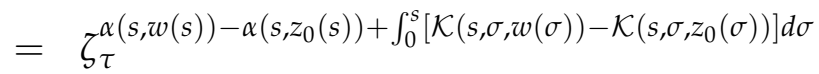

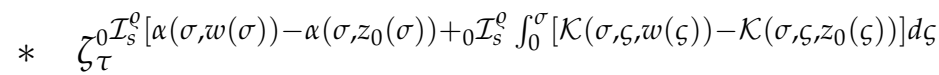

$$
\begin{aligned}
& \geq \psi_{j \frac{\tau}{2 \mathrm{~V}\left\{\ell_{1}, \ell_{2} \ell_{3}\right\}}}^{S} * \psi_{j \frac{\tau}{2 \ell_{4} \mathrm{~V}\left\{\ell_{1}, \ell_{2} \ell_{3}\right\}}}^{S} \\
& \geq \psi_{J \frac{\tau}{2 \bigvee\left\{\ell_{1}, \ell_{2} \ell_{3}\right\}\left(1+\ell_{4}\right)}}^{S},
\end{aligned}
$$

which implies that $\delta\left(Y(w), z_{0}\right) \leq \frac{2 \bigvee\left\{\ell_{1}, \ell_{2} \ell_{3}\right\}\left(1+\ell_{4}\right)}{j}<\infty$, hence $z_{0} \in B^{*}$. 
Corollary 1. Let $(\mathbb{R}, \zeta, *)$ be an $M B$-space and $\ell_{1}, \ell_{2}, \ell_{3}, \ell_{4}$ and $T$ be a positive constant such that $\bigvee\left\{\ell_{1}, \ell_{2} \ell_{3}, \ell_{1} \ell_{4}, \ell_{2} \ell_{3} \ell_{4}\right\}<0.5$. Assume that the continuous mappings $\alpha:[0, T] \times \mathbb{R} \rightarrow \mathbb{R}$, $\mathcal{K}:[0, T] \times[0, T] \times \mathbb{R} \rightarrow \mathbb{R}$ with DDM-valued $\psi:[0, T] \rightarrow S^{+}$satisfying

$$
\begin{gathered}
\zeta_{\tau}^{\alpha(s, u(s))-\alpha(s, v(s))} \geq \zeta_{\frac{\tau}{\ell_{1}}}^{u(s)-v(s),} \\
\zeta_{\tau}^{\mathcal{K}(s, \sigma, u(\sigma))-\mathcal{K}(s, \sigma, v(\sigma))} \geq \zeta_{\frac{\tau}{\ell_{2}}}^{u(\sigma)-v(\sigma)}, \sigma \leq s, \\
\inf _{\rho \in[0, T]} \psi_{\tau}^{\rho} \geq\left(\mathbf{W}_{\alpha, \beta}\right)_{\frac{T \tau}{\ell_{3}}}^{s},
\end{gathered}
$$

and

$$
\zeta_{\tau}^{u(s)} \geq\left(\mathbf{W}_{\alpha, \beta}\right)_{\tau}^{s}, \text { implies that } \zeta_{\tau}^{0 \mathcal{I}_{s}^{\varrho} u(\sigma) d \sigma} \geq\left(\mathbf{W}_{\alpha, \beta}\right)_{\frac{\tau}{\ell_{4}}}^{s}
$$

for every $s \in[0, T], u, v:[0, T] \rightarrow \mathbb{R}$ and $\tau \in \mathbb{J}^{\circ}$. Let $w:[0, T] \rightarrow \mathbb{R}$ be a differentiable function satisfying

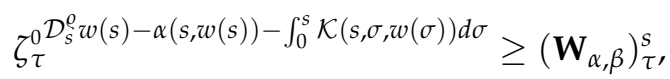

for every $s \in[0, T]$ and $\tau \in \mathbb{J}^{\circ}$. Thus, there is a unique differentiable function $w_{0}:[0, T] \rightarrow \mathbb{R}$ such that

$$
{ }_{0} \mathcal{D}_{s}^{\varrho} w_{0}(s)=\alpha\left(s, w_{0}(s)\right)+\int_{0}^{s} \mathcal{K}\left(s, \sigma, w_{0}(\sigma)\right) d \sigma
$$

and

$$
\zeta_{\tau}^{0} \mathcal{D}_{s}^{\rho} w(s)-{ }_{0} \mathcal{D}_{s}^{\rho} w_{0}(s) * \zeta_{\tau}^{w(s)-w_{0}(s)} \geq\left(\mathbf{W}_{\alpha, \beta}\right)_{\frac{\left(1-2 \bigvee\left\{\ell_{1}, \ell_{2} \ell_{3}, \ell_{1} \ell_{4}, \ell_{2} \ell_{3} \ell_{4}\right\}\right) \tau}{\mathrm{V}\left\{1, \ell_{4}\right\}}},
$$

for every $s \in[0, T]$ and $\tau \in \mathbb{J}^{\circ}$.

Proof. Put $\psi=\left(\mathbf{W}_{\alpha, \beta}\right)$ and apply Theorem 3 .

\section{Random Stability of Riemann-Liouville Fractional Volterra Integral Equation}

Consider the Riemann-Liouville fractional Volterra integral equation

$$
u(s)=\alpha(s, u(s))+{ }_{0} \mathcal{I}_{s}^{\varrho} \mathcal{K}(s, \sigma, u(\sigma)),
$$

where $\varrho \in \mathbb{I}^{\circ}, \alpha:[0, T] \times W \rightarrow W, \mathcal{K}:[0, T] \times[0, T] \times W \rightarrow W$. In this section, we study random Wright stability of (42).

Theorem 4. Suppose that $(W, \zeta, *)$ is an $M B$-space and $\ell_{1}, \ell_{2}, \ell_{3}, \ell_{4}$ and $T$ are positive constants such that $\bigvee\left\{\ell_{1}, \ell_{2} \ell_{3}, \ell_{1} \ell_{4}, \ell_{2} \ell_{3} \ell_{4}\right\}<0.5$. Assume the continuous mappings $\alpha:[0, T] \times W \rightarrow W$, $\mathcal{K}:[0, T] \times[0, T] \times W \rightarrow W$ with DDM-valued $\psi:[0, T] \rightarrow S^{+}$satisfying (35)-(38).

Let $w:[0, T] \rightarrow W$ be a differentiable function satisfying

$$
\zeta_{\tau}^{w(s)-\alpha(s, w(s))-{ }_{0} \mathcal{I}_{s}^{\varrho} \mathcal{K}(s, \sigma, w(\sigma))} \geq \psi_{\tau}^{s}
$$


for every $s \in[0, T]$ and $\tau \in \mathbb{J}^{\circ}$. Thus, we can find a unique differentiable function $w_{0}:[0, T] \rightarrow W$ such that

$$
w_{0}(s)=\alpha\left(s, w_{0}(s)\right)+{ }_{0} \mathcal{I}_{s}^{\varrho} \mathcal{K}\left(s, \sigma, w_{0}(\sigma)\right)
$$

and

$$
\zeta_{\tau}^{w(s)-w_{0}(s)} \geq \psi_{\frac{\left(1-2 \bigvee\left\{\ell_{1}, \ell_{2} \ell_{3}, \ell_{1} \ell_{4}, \ell_{2} \ell_{3} \ell_{4}\right\}\right) \tau}{V\left\{1, \ell_{4}\right\}}}^{s}
$$

for every $s \in[0, T]$ and $\tau \in \mathbb{J}^{\circ}$.

Proof. We set

$$
B:=\{u:[0, T] \rightarrow W, u \text { as differentiable }\}
$$

and define

$$
\delta(u, v)=\inf \left\{\mu \in \mathbb{J}^{\circ}: \zeta_{\tau}^{u(s)-v(s)} \geq \psi_{\frac{\tau}{\mu}}^{s}, \forall u, v \in B, s \in[0, T], \tau \in \mathbb{J}^{\circ}\right\} .
$$

Theorem 2 guarantees that $(B, \delta)$ is a complete $\mathbb{J}^{\bullet}$-valued metric space.

Consider $Y$ from $B$ to $B$ by

$$
\mathrm{Y}(u(s))=\alpha(\sigma, u(\sigma))+{ }_{0} \mathcal{I}_{s}^{\varrho}\left(\int_{0}^{\sigma} \mathcal{K}(\sigma, \varsigma, u(\varsigma)) d \varsigma\right),
$$

where $\varrho \in \mathbb{I}^{\circ}, \alpha:[0, T] \times W \rightarrow W, \mathcal{K}:[0, T] \times[0, T] \times W \rightarrow W$. We show that $\mathrm{Y}$ is a strictly contractive mapping. Let $u, v \in B, \mu \in \mathbb{J}^{\circ}$ and $\delta(u, v)<v$; then, we have

$$
\zeta_{v \tau}^{u(s)-v(s)} \geq \psi_{\tau}^{s}, \forall u, v \in B, s \in[0, T], \tau \in \mathbb{J}^{\circ} .
$$

From (MN2) and (MN3) of Definition 2, (35)-(38) and (46), we get

$$
\begin{aligned}
& \zeta_{2 v \tau}^{\mathrm{Y}(u(s))-\mathrm{Y}(v(s))} \\
& =\zeta_{2 v \tau}^{\alpha(s, u(s))-\alpha(s, v(s))]++_{0} \mathcal{I}_{s}^{Q}[\mathcal{K}(s, \sigma, u(\sigma))-\mathcal{K}(s, \sigma, v(\sigma))] d \sigma} \\
& \geq \zeta_{v \tau}^{\alpha(s, u(s))-\alpha(s, v(s))} * \zeta_{v \tau}^{\int_{0}^{s}[\mathcal{K}(s, \sigma, u(\sigma))-\mathcal{K}(s, \sigma, v(\sigma))] d \sigma} \\
& \geq \psi_{\frac{\tau}{\ell_{1}}}^{s} * \psi_{\frac{\tau}{\ell_{2} \ell_{4}}}^{s} \\
& \geq \psi_{\frac{\tau}{\mathrm{V}\left\{\ell_{1}, \ell_{2} \ell_{4}\right\}}}{ }^{\prime}
\end{aligned}
$$

and so

$$
\zeta_{v \tau}^{\mathrm{Y}(u(s))-\mathrm{Y}(v(s))} \geq \psi_{\frac{\tau}{2 \mathrm{~V}\left\{\ell_{1}, \ell_{2} \ell_{4}\right\}}},
$$

for every $s \in[0, T]$ and $\tau \in \mathbb{J}^{\circ}$. Consequently,

$$
\delta(\mathrm{Y}(u), \mathrm{Y}(v)) \leq 2 \bigvee\left\{\ell_{1}, \ell_{2} \ell_{4}\right\} v,
$$

and so

$$
\delta(\mathrm{Y}(u), \mathrm{Y}(v)) \leq 2 \bigvee\left\{\ell_{1}, \ell_{2} \ell_{4}\right\} \delta(u, v)
$$

Thus, $\mathrm{Y}$ is strictly contractive with Lipschitz constant $2 \bigvee\left\{\ell_{1}, \ell_{2} \ell_{4}\right\}$. 
Letting $w \in B$, we prove that $\delta(Y(w), w)<\infty$. In addition, (53) implies that

$$
\begin{aligned}
\zeta_{\tau}^{Y(w(s))-w(s)} & =\zeta_{\tau}^{\alpha(s, w(s))+{ }_{0} \mathcal{I}_{s}^{\rho} \mathcal{K}(s, \sigma, w(\sigma)) d \sigma-w(s)} \\
& \geq \psi_{\tau}^{s}
\end{aligned}
$$

for every $\tau \in \mathbb{J}^{\circ}$. Thus, $\delta(\mathrm{Y}(w), w)<1$.

By Theorem 1, there is $w_{0}$ in $B$ such that

(1) $w_{0}$ is a fixed point of $Y$, i.e.,

$$
\begin{aligned}
w_{0}(s) & =\mathrm{Y}\left(w_{0}(s)\right) \\
& =\alpha\left(\sigma, w_{0}(\sigma)\right)+{ }_{0} \mathcal{I}_{s}^{\varrho}\left(\mathcal{K}\left(\sigma, \varsigma, w_{0}(\varsigma)\right) d \varsigma\right),
\end{aligned}
$$

which is unique in the set

$$
B^{*}=\{u \in B: \delta(\mathrm{Y}(w), u)<\infty\} .
$$

(2) $\delta\left(Y^{k}(w), w_{0}\right) \rightarrow 0$ as $n \rightarrow \infty$;

(3) $\delta\left(w, w_{0}\right) \leq \frac{1}{1-2 \bigvee\left\{\ell_{1}, \ell_{2} \ell_{4}\right\}} \delta(\mathrm{Y}(w), w) \leq \frac{1}{1-2 \bigvee\left\{\ell_{1}, \ell_{2} \ell_{4}\right\}}$, which implies that

$$
\zeta_{\tau}^{w(s)-w_{0}(s)} \geq \psi_{\left(1-2 \bigvee\left\{\ell_{1}, \ell_{2} \ell_{4}\right\}\right) \tau^{\prime}}^{s}
$$

for every $s \in[0, T]$ and $\tau \in \mathbb{J}^{\circ}$. By the same method of the proof of Theorem 3, we can show that $B^{*}=B$.

Corollary 2. Suppose that $(\mathbb{R}, \zeta, *)$ is an $M B$-space and $\ell_{1}, \ell_{2}, \ell_{3}, \ell_{4}$ and $T$ are positive constants such that $\bigvee\left\{\ell_{1}, \ell_{2} \ell_{3}, \ell_{1} \ell_{4}, \ell_{2} \ell_{3} \ell_{4}\right\}<0.5$. Assume that the continuous mappings $\alpha:[0, T] \times \mathbb{R} \rightarrow$ $\mathbb{R}, \mathcal{K}:[0, T] \times[0, T] \times \mathbb{R} \rightarrow \mathbb{R}$ with DDM-valued $\psi:[0, T] \rightarrow S^{+}$satisfying (35)-(38).

Let $w:[0, T] \rightarrow \mathbb{R}$ be a differentiable function satisfying

$$
\zeta_{\tau}^{w(s)-\alpha(s, w(s))-{ }_{0} \mathcal{I}_{s}^{\varrho} \mathcal{K}(s, \sigma, w(\sigma))} \geq\left(\mathbf{W}_{\alpha, \beta}\right)_{\tau}^{s},
$$

for every $s \in[0, T]$ and $\tau \in \mathbb{J}^{\circ}$. Thus, we can find a unique differentiable function $w_{0}:[0, T] \rightarrow \mathbb{R}$ such that

$$
w_{0}(s)=\alpha\left(s, w_{0}(s)\right)+{ }_{0} \mathcal{I}_{s}^{\varrho} \mathcal{K}\left(s, \sigma, w_{0}(\sigma)\right),
$$

and

$$
\zeta_{\tau}^{w(s)-w_{0}(s)} \geq\left(\mathbf{W}_{\alpha, \beta}\right)_{\frac{\left(1-2 \bigvee\left\{\ell_{1}, \ell_{2} \ell_{3}, \ell_{1} \ell_{4}, \ell_{2} \ell_{3} \ell_{4}\right\}\right) \tau}{V}}^{V_{\left\{1, \ell_{4}\right\}}}
$$

for every $s \in[0, T]$ and $\tau \in \mathbb{J}^{\circ}$.

Proof. Put $\psi=\left(\mathbf{W}_{\alpha, \beta}\right)$ and apply Theorem 4 .

\section{Applications, Random Wright Stability}

Now, as applications, we study the concept of random Wright stability for some fractional equations.

Example 1. Assume that $(\mathbb{R}, \zeta, *)$ is an MB-space. Consider $u, v:[0, T] \rightarrow \mathbb{R}$ and define $\alpha(s, u(s))=\ell_{1} u(s)$. Let $\theta \in \mathcal{W}^{1,1}\left(\mathbb{J}^{\circ}, \mathbb{R}\right)$, in which $\mathcal{W}^{1,1}$ is the Sobolev space, define $\mathcal{K}$ : $[0, T] \times[0, T] \times \mathbb{R} \rightarrow \mathbb{R}$ as $\mathcal{K}(s, \sigma, u(\sigma))=\theta(s-\sigma) u(\sigma)$ for every $s \in[0, T]$ and $\sigma \leq s$. 
Consequently, we have

$$
\begin{aligned}
\zeta_{\tau}^{\alpha(s, u(s))-\alpha(s, v(s))} & =\zeta_{\tau}^{\ell_{1} u(s)-\ell_{1} v(s)} \\
& =\zeta_{\frac{\tau}{\ell_{1}}}^{u(s)-v(s)}, \\
\zeta_{\tau}^{\mathcal{K}(s, \sigma, u(\sigma))-\mathcal{K}(s, \sigma, v(\sigma))} & =\zeta_{\tau}^{\theta(s-\sigma)[u(\sigma)-v(\sigma)]} \\
& \geq \zeta_{\frac{\tau}{|\theta(s-\sigma)|}}^{u(\sigma)-v(\sigma)} \\
& \geq \zeta_{\frac{\tau}{K}}^{u(\sigma)-v(\sigma)},
\end{aligned}
$$

for some $K \in \mathbb{J}^{\circ}$. Let DDM-valued $\mathbf{W}_{\alpha, \beta}:[0, T] \rightarrow S^{+}$satisfying (37) and (38).

Let $w:[0, T] \rightarrow \mathbb{R}$ be a differentiable function satisfying

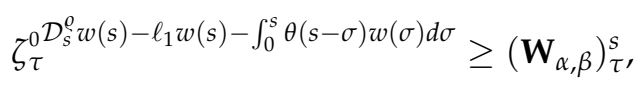

for every $s \in[0, T]$ and $\tau \in \mathbb{J}^{\circ}$. Now, Theorem 3 implies that, if $\bigvee\left\{\ell_{1}, K \ell_{3}, \ell_{1} \ell_{4}, K \ell_{3} \ell_{4}\right\}<0.5$, there is a unique differentiable function $w_{0}:[0, T] \rightarrow \mathbb{R}$ such that

$$
{ }_{0} \mathcal{D}_{s}^{\varrho} w_{0}(s)=\ell_{1} w_{0}(s)+\int_{0}^{s} \theta(s-\sigma) w(\sigma) d \sigma
$$

and

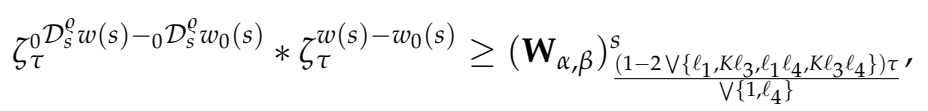

for every $s \in[0, T]$ and $\tau \in \mathbb{J}^{\circ}$.

Example 2. Assume that $(\mathbb{R}, \zeta, *)$ is an $M B$-space. Consider $u, v:[0, T] \rightarrow \mathbb{R}$ and define $\alpha(s, u(s))=\ell_{1} u(s)$. Let $\theta \in \mathcal{W}^{1,1}\left(\mathbb{J}^{\circ}, \mathbb{R}\right)$, in which $\mathcal{W}^{1,1}$ is the Sobolev space, and define $\mathcal{K}:[0, T] \times[0, T] \times \mathbb{R} \rightarrow \mathbb{R}$ as $\mathcal{K}(s, \sigma, u(\sigma))=\theta(s-\sigma) u(\sigma)$ for every $s \in[0, T]$ and $\sigma \leq s$ satisfying (57).

Let DDM-valued $\mathbf{W}_{\alpha, \beta}:[0, T] \rightarrow S^{+}$satisfying (37) and (38).

Let $w:[0, T] \rightarrow \mathbb{R}$ be a differentiable function satisfying

$$
\zeta_{\tau}^{w(s)-\ell_{1} w(s)-{ }_{0} \mathcal{I}_{s}^{\varrho} \theta(s-\sigma) w(\sigma) d \sigma} \geq\left(\mathbf{W}_{\alpha, \beta}\right)_{\tau}^{s}
$$

for every $s \in[0, T]$ and $\tau \in \mathbb{J}^{\circ}$. Now, Theorem 4 implies that, if $\bigvee\left\{\ell_{1}, K \ell_{4}\right\}<0.5$, there is a unique differentiable function $w_{0}:[0, T] \rightarrow \mathbb{R}$ such that

$$
w_{0}(s)=\ell_{1} w_{0}(s)+{ }_{0} \mathcal{I}_{s}^{\varrho} \theta(s-\sigma) w(\sigma) d \sigma,
$$

and

$$
\zeta_{\tau}^{w(s)-w_{0}(s)} \geq\left(\mathbf{W}_{\alpha, \beta}\right)_{\frac{\left(1-2 \bigvee\left\{\ell_{1}, K \ell_{4}\right\}\right) \tau}{}}^{{\mathcal{V}\left\{1, \ell_{4}\right\}}^{\prime}}
$$

for every $s \in[0, T]$ and $\tau \in \mathbb{J}^{\circ}$.

\section{Conclusions}

We considered a class of random control functions. By a method from Mihet and Radu emanating from the alternative fixed point theorem and random controllers, we stabilized some fractional equations in MB-spaces in the sense of Wright. 
Author Contributions: Data curation, R.S.; Formal analysis, R.M.; Methodology, R.S.; Project administration, R.M. and R.S.; Resources, R.S. Both authors have read and agreed to the published version of the manuscript.

Funding: This research received no external funding.

Institutional Review Board Statement: Not applicable.

Informed Consent Statement: Not applicable.

Data Availability Statement: Not applicable.

Acknowledgments: The authors are thankful for the area editor and the reviewers for giving valuable comments and suggestions. The work of the first author in this paper was supported by grant APVV18-0052.

Conflicts of Interest: The authors declare no potential conflict of interest.

\section{References}

1. Kilbas, A.A.; Srivastava, H.M.; Trujillo, J.J. Theory and Applications of Fractional Differential Equations; North-Holland Mathematics Studies; Elsevier Science B.V.: Amsterdam, The Netherlands, 2006; Volume 204.

2. Povstenko, Y. Linear Fractional Diffusion-Wave Equation for Scientists and Engineers; Springer: Cham, Switzerland, 2015.

3. Podlubny, I. Fractional Differential Equations. An Introduction to Fractional Derivatives, Fractional Differential Equations, to Methods of Their Solution and Some of Their Applications; Mathematics in Science and Engineering; Academic Press, Inc.: San Diego, CA, USA, 1999; Volume 198.

4. Saadati, R. Nonlinear contraction and fuzzy compact operator in fuzzy Banach algebras. Fixed Point Theory 2019, $20,289-297$. [CrossRef]

5. Schweizer, B.; Sklar, A. North-Holland Series in Probability and Applied Mathematics; North-Holland Publishing Co.: New York, NY, USA, 1983.

6. S̆ Serstnev, A.N. On the notion of a random normed space. Dokl. Akad. Nauk SSSR 1963, 149, 280-283. (In Russian)

7. Hadzic, O.; Pap, E. Fixed Point Theory in Probabilistic Metric Spaces; Mathematics and Its Applications; Kluwer Academic Publishers: Dordrecht, The Netherlands, 2001; Volume 536.

8. Klement, E.P.; Mesiar, R.; Pap, E. Triangular Norms; Trends in Logic-Studia Logica Library; Kluwer Academic Publishers: Dordrecht, The Netherlands, 2000; Volume 8.

9. Madadi, M.; O’Regan, D.; Rassias, T.M.; Saadati, R. Best approximation of $\kappa$-random operator inequalities in matrix MB-algebras. J. Inequal. Appl. 2021, 2021, 14. [CrossRef]

10. Madadi, M.; Saadati, R.; Park, C.; Rassias, J.M. Stochastic Lie bracket (derivation, derivation) in MB-algebras. J. Inequal. Appl. 2020, 2020, 15. [CrossRef]

11. Cădariu, L.; Radu, V. Fixed points and the stability of Jensen's functional equation. JIPAM. J. Inequal. Pure Appl. Math. $2003,4,7$.

12. Diaz, J.B.; Margolis, B. A fixed point theorem of the alternative, for contractions on a generalized complete metric space. Bull. Am. Math. Soc. 1986, 74, 305-309. [CrossRef]

13. Golet, I. Some remarks on functions with values in probabilistic normed spaces. Math. Slovaca 2007, 57, 259-270. [CrossRef]

14. Srivastava, H.M.; Manocha, H.L. A Treatise on Generating Functions; Ellis Horwood Series: Mathematics and Its Applications; Ellis Horwood Ltd.: Chichester, UK; John Wiley \& Sons, Inc.: New York, NY, USA, 1984.

15. Wright, E.M. The generalized Bessel function of order greater than one. Q. J. Math. Oxf. Ser. 1940, 11, 36-48. [CrossRef]

16. Radu, V. The fixed point alternative and the stability of functional equations. Fixed Point Theory 2003, 4, 91-96.

17. Miheț, D.; Radu, V. On the stability of the additive Cauchy functional equation in random normed spaces. J. Math. Anal. Appl. 2008, 343, 567-572. [CrossRef]

18. Agarwal R.P.; Hristova, S.; O'Regan, D. Lyapunov functions to Caputo reaction-diffusion fractional neural networks with time-varying delays. J. Math. Comput. Sci. 2018, 18, 328-345. [CrossRef]

19. Ahmed, H.M.; El-Borai, M.M.; El-Owaidy, H.M.; Ghanem, A.S. Null controllability of fractional stochastic delay integrodifferential equations. J. Math. Comput. Sci. 2019, 19, 143-150. [CrossRef]

20. Khan, O.; Araci, S.; Saif, M. Fractional calculus formulas for Mathieu-type series and generalized Mittag-Leffler function. J. Math. Comput. Sci. 2020, 20, 122-130. [CrossRef]

21. Chaharpashlou, R.; Saadati, R.; Atangana, A. Ulam-Hyers-Rassias stability for nonlinear $\Psi$-Hilfer stochastic fractional differential equation with uncertainty. Adv. Differ. Equ. 2020, 2020, 10. [CrossRef]

22. Chaharpashlou, R.; Saadati, R. Best approximation of a nonlinear fractional Volterra integro-differential equation in matrix MB-space. Adv. Differ. Equ. 2021, 2021, 12. [CrossRef]

23. Eidinejad, Z.; Saadati, R.; de la Sen, M. Radu-Mihet method for the existence, uniqueness, and approximation of the $\psi$-Hilfer fractional equations by matrix-valued fuzzy controllers. Axioms 2021, 10, 63. [CrossRef]

24. El-hady, E.; Ogrekci, S. On Hyers-Ulam-Rassias stability of fractional differential equations with Caputo derivative. J. Math. Comput. Sci. 2021, 22, 325-332. [CrossRef] 
25. El-Ajou, A. Taylor's expansion for fractional matrix functions: Theory and applications. J. Math. Comput. Sci. 2020, 21, 1-17. [CrossRef]

26. El-Sayed, A.M.A.; Al-Issa, S.M. Existence of integrable solutions for integro-differential inclusions of fractional order; coupled system approach. J. Nonlinear Sci. Appl. 2020, 13, 180-186. [CrossRef]

27. El-Moneam, M.A.; Ibrahim, T.F.; Elamody, S. Stability of a fractional difference equation of high order. J. Nonlinear Sci. Appl. 2019, 12, 65-74. [CrossRef]

28. Sene, N. Global asymptotic stability of the fractional differential equations. J. Nonlinear Sci. Appl. 2020, 13, 171-175. [CrossRef]

29. Sene, N. Stability analysis of the generalized fractional differential equations with and without exogenous inputs. J. Nonlinear Sci. Appl. 2019, 12, 562-572. [CrossRef]

30. Youssef, M.I. Caputo-Katugampola fractional Volterra functional differential equations with a vanishing lag function. J. Nonlinear Sci. Appl. 2020, 13, 293-302. [CrossRef]

31. Da Sousa, J.V.C.; Fabio, G.R.; de Oliveira, E.C. Stability of the fractional Volterra integro-differential equation by means of $\Psi$-Hilfer operator. Math. Meth. Appl. Sci. 2019, 42, 3033-3043. [CrossRef]

32. Cădariu, L.; Radu, V. On the stability of the Cauchy functional equation: A fixed point approach. Grazer Math. Ber. 2004, $346,43-52$.

33. Cădariu, L.; Radu, V. Fixed point methods for the generalized stability of functional equations in a single variable. Fixed Point Theory Appl. 2008, 2008, 749392. [CrossRef]

34. Rezaei Aderyani, S.; Saadati, R. Best approximations of the $\phi$-Hadamard fractional Volterra integro-differential equation by matrix valued fuzzy control functions. Adv. Differ. Equ. 2021, 2021, 21. [CrossRef] 